\title{
[gw22-e0570] EXTRACORPOREAL CARDIAC SHOCK WAVE THERAPY IMPROVED MYOCARDIAL MICRO- VASCULAR CIRCULATION AFTER ACUTE MYOCARDIAL INFARCTION AT EARLY STAGE IN PIGS
}

Tao Siming', Guo Tao², Wan Yu ${ }^{1}$ The 4th Affiliated Hospital Of Kunming Medical College, ${ }^{2}$ The No.1 Affiliated Hospital of Kunming Medical College, Kunming, China

10.1136/heartjnl-2011-300867.208

Objective In the clinical prospect, the goal for the treatment of ischemic heart disease should include not only enhancement of angiogenesis but also reconstruction of region cardiomyocytes micro-vascular circulation and recovery of ischemia induced myocardial dysfunction. This experimental study aims to investigate the effects of CSWT on improving myocardial micro-vascular circulation after acute myocardial infarction at the early stage.

Methods Thirty domestic miniature pigs were enrolled this study. The methods of grouping and treatment were same as those in part II experiment. At 30 days, the number of endothelium cell, capillary density and collateral vessel Rentrop score were measured and evaluated by immunohistochemisty and coronary artery angiography, and then compared among every group, respectively. Results Shock wave treatment up-regulated the mRNA expression of VEGF in model of acute myocardial infarction. At the same time, the number of capillaries was significantly higher in the shock wave group than that of pseudo-CSWT group (1856 \pm 78 vs $\left.837 \pm 54 / \mathrm{mm}^{2}, \mathrm{p}<0.0001\right)$. There was no significant difference between pseudo-CSWT group and blank control group $\left(837 \pm 54\right.$ vs $569 \pm 32 / \mathrm{mm}^{2}$, $\mathrm{p}>0.05$ ); Furthermore, these improving effects in subgroup of prolonged duration CSWT were better than other two subgroups. Compared with pseudo-CSWT group, collateral vessel Rentrop score was significantly higher in CSWT group $(2.05 \pm 0.11$ vs $0.98 \pm 0.09, \mathrm{p}=0.03)$.

Conclusions Extracorporeal cardiac shock wave therapy could not only effectively induce angiogenesis and up-regulate the expression of angiogenic factor, but also, result in an improvement in reconstructing micro-vascular circulation of ischemia myocardial, especially, prolonged duration CSWT in early stage of acute myocardial infarction could improve myocardial micro-vascular circulation. This may be one of the most important mechanisms for CSWT angiogenesis strategy in treatment of human ischemia heart disease in early stage. 